\title{
Erratum to: General Non-Existence Theorem for Phase Transitions in One-Dimensional Systems with Short Range Interactions, and Physical Examples of Such Transitions
}

\author{
José A. Cuesta · Angel Sánchez
}

Published online: 7 November 2009

(C) Springer Science+Business Media, LLC 2009

\section{Erratum to: J Stat Phys (2004) 115: 869-893 DOI 10.1023/B:JOSS.0000022373.63640.4e}

We have found an erratum in our non-existence theorem (Theorem 5): There is one hypothesis missing in the text (analyticity) which is needed to apply Theorem 4 in the proof. In addition, we have noticed that, as formulated in the paper, the Theorem is too restrictive, because the positivity hypothesis is needed only on $\mathbb{R}^{+}$.

Taking these two issues into account, Theorem 5 should read as follows (changes in straight font):

Theorem 5 (Nonexistence of Phase Transitions) Let $\mathbf{T}(\beta)$ be a compact, irreducible linear operator on the Banach lattice $E$ for all $\beta \in \Omega$, a complex neighborhood of $\mathbb{R}^{+}$. Assume further that $\mathbf{T}(\beta)$ is analytic in $\Omega$ either in the strong or the weak convergence sense, and positive on $\mathbb{R}^{+}$. Let $\lambda_{\max }(\beta)$ and $\mathbf{P}_{\max }(\beta)$ be, respectively, the maximum eigenvalue of $\mathbf{T}(\beta)$ and the projector on its corresponding eigenspace. Let $\varphi(\cdot)$ be a real, linear functional on the space of bounded, linear operators on $E$ such that $\varphi\left(\mathbf{P}_{\max }(\beta)\right) \neq 0$. Then

$$
\lim _{N \rightarrow \infty} \frac{1}{N} \ln \mathcal{Z}_{N}=-\ln \lambda_{\max }(\beta)
$$

is an analytic function on $\mathbb{R}^{+}$, where $\mathcal{Z}_{N}$ is given by equation (25).

The online version of the original article can be found under doi:10.1023/B:JOSS.0000022373.63640.4e.

J.A. Cuesta $(\bowtie) \cdot$ A. Sánchez

Grupo Interdisciplinar de Sistemas Complejos (GISC) and Departamento de Matemáticas, Universidad Carlos III de Madrid, Avenida de la Universidad 30, 28911 Leganés, Madrid, Spain

e-mail: cuesta@math.uc3m.es

A. Sánchez

e-mail: anxo@math.uc3m.es 
The proof holds as is in the paper, with a minor modification on the last paragraph, which should read (changes in italics):

Now, $\mathbf{T}(\beta)$ fulfills the hypothesis of Theorem 3, thus for all $\beta \in \mathbb{R}^{+}, \lambda_{\max }(\beta)>0$ has multiplicity one and is isolated. By continuity, so it is in a complex neighborhood of $\mathbb{R}^{+}$. Then taking $\Sigma=\left\{\lambda_{\max }(\beta)\right\}$ in Theorem 4 it follows that this eigenvalue is an analytic function in $\beta>0$ and the proof is complete.

All the conclusions and discussions of the paper are unaffected by these technical points, needed only for mathematical accuracy. 